\title{
One in a billion: a patient with Marfan syndrome and familial hypocalciuric hypercalcaemia
}

\author{
Su Ann Tee1, Paul Brennan² and Anna L Mitchell1 \\ 'Department of Endocrinology, Newcastle upon Tyne Hospitals NHS Foundation Trust, Newcastle, UK and \\ 2Departmentof Northern Genetics Service, Newcastle upon Tyne Hospitals NHS Foundation Trust, Newcastle, UK
}

Correspondence should be addressed to Su Ann Tee

E-mail

suann.tee@nhs.net

\begin{abstract}
Summary
Marfan syndrome is an autosomal dominant multisystem disorder that has an estimated incidence of 1 in 5000 . It is caused by mutations in the FBN1 gene, which encodes the extracellular matrix protein type 1 fibrillin. Familial hypocalciuric hypercalcaemia $(\mathrm{FHH})$, also inherited in an autosomal dominant pattern, is a rare benign disorder characterised by hypercalcaemia, hypocalciuria and relative hyperparathyroidism with normal or high plasma PTH levels, with an estimated incidence of between 1 in 10000 to 1 in 100 000. We report a unique case of a 26-year-old man referred for investigation of hypercalcaemia, who also had clinical features of Marfan syndrome but no previous genetic investigations. Calculated fractional urinary excretion of calcium was low (0.0005) following correction of vitamin D deficiency, raising the possibility of FHH. Genetic testing for Marfan syndrome and FHH, via a hyperparathyroidism multiplex gene panel test, revealed a novel truncating variant in the FBN1 gene (c.8481T>G; p.(Tyr2827Ter)), consistent with Marfan syndrome; and a pathogenic truncating variant in the CaSR gene (c.741dupT; p.[Asp248Ter]), which confirmed the diagnosis of FHH. The patient's mother was subsequently found to have mild hypercalcaemia (adjusted calcium $2.76 \mathrm{mmol} / \mathrm{L}$ ) and is also heterozygous for the same CaSR mutation. Genetic testing of his father confirmed the presence of the same FBN1 gene mutation. This case illustrates the importance of making robust diagnoses in the era of modern genomic medicine, confirming FHH as the cause of hypercalcaemia means that no treatment is warranted and the patient can be reassured.
\end{abstract}

\section{Learning points:}

- Familial hypocalciuric hypercalcaemia $(\mathrm{FHH})$ should always be excluded during the investigation of hypercalcaemia by measuring urinary calcium: creatinine clearance ratio.

- Diagnosing FHH is important as the condition is benign and misdiagnosing patients with primary hyperparathyroidism could potentially lead to unnecessary morbidity from parathyroid surgery.

- Genetic testing is increasingly available for a variety of inherited conditions including Marfan syndrome and FHH. Patients who present with clinical features suggestive of a particular genetic condition should undergo prompt, appropriate confirmatory testing wherever possible.

- Taking a thorough family history is vital when assessing patients presenting with endocrine conditions, as this could prompt cascade testing and appropriate genetic counselling where necessary. 


\section{Background}

We report a unique case of a patient diagnosed with two rare genetic conditions, inheriting Marfan syndrome from his father, and familial hypocalciuric hypercalcaemia separately from his mother. This is the first case to our knowledge, of a patient with both Marfan syndrome and FHH. Additionally, he was found to have a novel pathogenic variant in the FBN1 gene which has not been previously documented.

\section{Case presentation}

A 26-year-old man was referred to the Endocrinology service from primary care with hypercalcaemia. He had a longstanding clinical diagnosis of Marfan syndrome based on phenotypic features and a family history of Marfan syndrome in his father (no previous genetic confirmation) and paternal uncle. He was under regular cardiology review with annual echocardiograms which showed a mildly dilated aortic root ( $44 \mathrm{~mm}$ at the sinus of Valsalva) and mild left ventricular systolic dysfunction, treated with Losartan $50 \mathrm{mg}$ once daily and Bisoprolol $2.5 \mathrm{mg}$ once daily. He had bilateral moderate myopia (spherical equivalent objective refractive error $-5.25 \mathrm{D}$ in right eye; $-5.75 \mathrm{D}$ in left eye) and had a past history of a retinal tear, but otherwise, he was fit and well.

Hypercalcaemia with hyperparathyroidism was highlighted on routine blood tests requested in primary care as part of the investigation of persistent retching - the patient was referred to endocrinology with a provisional diagnosis of primary hyperparathyroidism (PHPT). The combination of skeletal features of Marfan syndrome and PHPT raised the possibility of MEN2 and therefore the referral was directed to the Joint Endocrine Genetics clinic where the patient was evaluated.

On examination, the patient was noted to be tall but with normal body proportions (height $192.5 \mathrm{~cm}$, weight $105.5 \mathrm{~kg}$, calculated BMI 28.5), and no convincing evidence of long bone overgrowth. He had a degree of kyphosis but not scoliosis. He did not have arachnodactyly and, in fact, had relatively small hands with bilateral flexion contractures affecting his fifth digits. He also did not exhibit any distinct facial features associated with Marfan syndrome such as dolichocephaly, enophthalmos, micrognathia or malar hypoplasia. Systemic examination was otherwise unremarkable. In particular, he did not have mucosal neuromas.

\section{Investigation}

Initial biochemical investigations showed an elevated adjusted calcium level of $2.68 \mathrm{mmol} / \mathrm{L}(2.2-2.6 \mathrm{mmol} / \mathrm{L})$, with a mildly elevated parathyroid hormone (PTH) level of $7.2 \mathrm{pmol} / \mathrm{L}$ (1.1-6.4 pmol/L), normal serum phosphate level of $0.96 \mathrm{mmol} / \mathrm{L}(0.80-1.50 \mathrm{mmol} / \mathrm{L})$ and a low vitamin D level of $37 \mathrm{nmol} / \mathrm{L}$ (>50 nmol/L).

Vitamin D supplementation with cholecalciferol was commenced at a dose of 800 units once daily. Following correction of vitamin D deficiency, he had persistent mild hypercalcaemia (adjusted calcium $2.74 \mathrm{mmol} / \mathrm{L}$, PTH 6.1 pmol/L, vitamin D $63 \mathrm{nmol} / \mathrm{L}$ ). Urinalysis, once vitamin D replete, showed urine calcium $0.2 \mathrm{mmol} / \mathrm{L}$, urine creatinine $14.6 \mathrm{mmol} / \mathrm{L}$, ratio 0.01 , and low calculated fractional urinary excretion of calcium (0.0005). On review of historical biochemistry records, the patient was noted to have relatively longstanding mild hypercalcaemia (2017 - $2.55 \mathrm{mmol} / \mathrm{L} ; 2016-2.62 \mathrm{mmol} / \mathrm{L}$ ), with a magnesium level at the upper limit of normal $(0.86 \mathrm{mmol} / \mathrm{L} ; 0.7-1.0$ $\mathrm{mmol} / \mathrm{L}$ ). The biochemical findings raised the possibility of familial hypocalciuric hypercalcaemia (FHH) and therefore dual genetic pathology.

Genetic testing for Marfan syndrome and FHH, via a hyperparathyroidism multiplex gene panel test, was undertaken with the patient's consent. This revealed a novel truncating variant in the $F B N 1$ gene $(c .8481 T>G$; p.(Tyr2827Ter)), consistent with Marfan syndrome. He was also found to have a pathogenic truncating variant in the CaSR gene (c.741dupT; p.[Asp248Ter]), which confirmed the diagnosis of FHH. Analysis of MEN1, CDKN1A, CDKN1B, CDKN2C and RET genes (exons 5, 7, 8, 10, 11, 13, 14-16) revealed no mutations.

\section{Outcome and follow-up}

The patient's mother was subsequently found to have mild hypercalcaemia (adjusted calcium $2.76 \mathrm{mmol} / \mathrm{L}$ ) and are also heterozygous for the same CaSR mutation. Genetic testing of his father confirmed the presence of the same FBN1 gene mutation (c.8481T>G [p.(Tyr2827Ter)]).

\section{Discussion}

Marfan syndrome is an autosomal dominant multisystem disorder that has an estimated incidence of 1 in 5000 (1). Antoine-Bernard Marfan, a paediatrician, first described this condition more than a century ago in a 5-year-old girl 
with long slender digits and other skeletal abnormalities. Since then, it has been established that the condition is caused by mutations in the FBN1 gene, which encodes the extracellular matrix protein type 1 fibrillin. Typical manifestations of Marfan syndrome include thoracic aortic aneurysm, ectopia lentis (dislocation of the ocular lens), and excessive long-bone overgrowth, resulting in disproportionate tall stature and rib overgrowth causing pectus deformity, often the most striking clinical sign. Myopia is extremely common in Marfan syndrome, and indeed myopia exceeding $-3.0 \mathrm{D}$ is one of the criteria included in the Ghent systemic score validated for clinical diagnosis (2). Thoracic aortic aneurysm and dissection have historically been recorded as the main causes of premature death, however, with the increasing success of aortic root replacement surgery, the life expectancy of patients with Marfan syndrome is now reported to be near-normal (3).

Our patient did not display many phenotypic features of Marfan syndrome, as described above. However, given the positive family history of Marfan syndrome in his father and uncle, there was a high index of suspicion that he could have a similar pathogenic mutation, prompting further investigation. This illustrates that the level of evidence required to make the diagnosis in a patient with a positive family history of Marfan syndrome (or indeed, alternative genetic disorders) is often lower than in a sporadic case. This principle is reflected in the revised Ghent diagnostic criteria (2010), whereby fewer clinical characteristics need to be present in an individual to confirm a diagnosis of Marfan syndrome, in the presence of a positive family history (2).

FHH, which is a benign autosomal dominant disorder characterised by hypercalcaemia, hypocalciuria and relative hyperparathyroidism with normal or high plasma PTH levels, has an estimated incidence of between 1 in 10000 and 1 in 100000 (4). Although the majority of patients with $\mathrm{FHH}$ are asymptomatic, it can also be associated with symptoms including muscle weakness, fatigue, arthralgia, increased thirst; in more severe cases, chondrocalcinosis and even nephrolithiasis have been observed. Case reports have linked FHH with acute pancreatitis but this is yet to be definitively established. Clues in the history that point to a diagnosis of FHH include longstanding, stable hypercalcaemia and a family history of hypercalcaemia inherited in an autosomal dominant pattern. It is important to distinguish FHH from primary hyperparathyroidism, as parathyroidectomy in FHH will not restore normocalcaemia.

It is sometimes difficult to distinguish $\mathrm{FHH}$ from primary hyperparathyroidism (PHPT) as there is a degree of overlap, both clinically and biochemically. However, there are numerous cases of morbidity related to unnecessary parathyroid surgery in patients with FHH. Therefore, it is imperative to clarify the diagnosis prior to referring for surgical intervention. It is reported that up to $9-23 \%$ of patients who underwent failed neck exploration for presumed PHPT were eventually diagnosed with FHH $(5,6)$. In our patient's case, accurate diagnosis of FHH and hence avoidance of unwarranted surgery was of utmost importance, as he would have been at higher risk of developing complications during general anaesthetic given the potential cardiovascular complications due to Marfan syndrome, and his reduced ventricular function.

The unique diagnoses of Marfan syndrome and FHH in our patient have also alerted us to be vigilant regarding symptoms of joint pain during a routine review. Patients with Marfan syndrome often develop joint pain and having co-existing FHH may place our patient at additional risk of chondrocalcinosis which also causes joint pain (although this is thankfully rare).

This patient's novel pathogenic FBN1 variant has been inherited from his father, while his CaSR gene mutation has been inherited from his mother. Therefore, assuming an incidence of Marfan syndrome of 1 in 5000 and an incidence of FHH of 1 in 50000 , the probability that one individual will inherit both conditions from his/her parents is 1 in a billion $(1 / 5000 \times$ $1 / 50000 \times 1 / 2 \times 1 / 2$ ).

In conclusion, we report the first case of FHH in an individual with Marfan syndrome and a novel pathogenic variant in the FBN1 gene. This case illustrates the importance of making robust diagnoses in the era of modern genomic medicine, confirming FHH as the cause of hypercalcaemia means that no treatment is warranted and the patient can be reassured.

\section{Declaration of interest}

The authors declare that there is no conflict of interest that could be perceived as prejudicing the impartiality of the research reported.

\section{Funding}

This research did not receive any specific grant from any funding agency in the public, commercial or not-for-profit sector.

\section{Patient consent}

The authors confirm that written informed consent was obtained from the patient for publication of the submitted article and accompanying images through his signature on their consent form. 


\section{Author contribution statement}

Dr SA Tee wrote the initial manuscript; Dr P Brennan was involved in reporting the genetic findings and contributed revisions to the manuscript; $\operatorname{Dr} A$ Mitchell was involved in the initial investigation, is responsible for ongoing care of the patient, and contributed to final revisions of the manuscript.

\section{References}

1 Judge DP \& Dietz HC. Marfan syndrome. Lancet 2005366 1965-1976. (https://doi.org/10.1016/S0140-6736(05)67789-6)

2 Loeys BL, Dietz HC, Braverman AC, Callewaert BL, De Backer J, Devereux RB, Hilhorst-Hofstee Y, Jondeau G, Faivre L, Milewicz DM, et al. The revised ghent nosology for the marfan syndrome. Journal of Medical Genetics 201047 476-485. (https://doi.org/10.1136/ jmg.2009.072785)
3 Silverman DI, Burton KJ, Gray J, Bosner MS, Kouchoukos NT, Roman MJ, Boxer M, Devereux RB \& Tsipouras P Life expectancy in the Marfan syndrome. American Journal of Cardiology 199575 157-160. (https://doi.org/10.1016/s0002-9149(00)80066-1)

4 Lee JY \& Shoback DM Familial hypocalciuric hypercalcaemia and related disorders. Best Practice and Research. Clinical Endocrinology and Metabolism 201832 609-619. (https://doi.org/10.1016/j. beem.2018.05.004)

5 Christensen SE, Nissen PH, Vestergaard P \& Mosekilde L Familial hypocalciuric hypercalcaemia: a review. Current Opinion in Endocrinology, Diabetes, and Obesity 201118 359-370. (https://doi. org/10.1097/MED.0b013e32834c3c7c)

6 Marx SJ, Stock JL, Attie MF, Downs RW, Gardner DG, Brown EM, Spiegel AM, Doppman JL \& Brennan MF Familial hypocalciuric hypercalcaemia: recognition among patients referred after unsuccessful parathyroid exploration. Annals of Internal Medicine 1980 92 351-356. (https://doi.org/10.7326/0003-4819-92-3-351)

Received in final form 6 April 2021

Accepted 13 May 2021 